\title{
Short Communication \\ Total RNA isolation and cDNA synthesis from Bixa orellana bark
}

\author{
Santosh $\mathrm{P}^{1}$ and Suresh B. Arakera ${ }^{2}$ \\ ${ }^{1}$ Division of Molecular Entomology, ICAR- National Bureau of Agricultural Insect \\ Resources (NBAIR), Bengaluru-560024, Karnataka, India \\ ${ }^{2}$ Department of Marine Biology, Karnatak University Post Graduate Center, KodibagKarwar- \\ 581303, Karnataka, India. \\ *Corresponding author E-mail: santoshsarathy@gmail.com
}

Annatto is one of the important natural food colourant widely used in dairy industry. Quality RNA in large quantity is often required in the analysis of gene expression. RNA extraction from samples collected from woody plants is generally complex, and becomes the main limitation to study gene expression, particularly in crops like Bixa orellana. Standard RNA extraction protocols are time consuming, laborious and cannot be adapted for high throughput functional analysis. Therefore a simple and effective protocol for extraction of high quality total RNA from bark tissue of woody stem was achieved using the RNeasy plant mini kit (Qiagen, USA). The extracted RNA was successfully converted into double-stranded cDNA using the SMATer cDNA synthesis kit (Clontech, USA) which is based on the Switching Mechanism At $5^{\prime}$ End of $\underline{R} N A$ Transcript (SMART) technology. The integrity of the total RNA used for synthesizing double stranded cDNA was assessed agarose gel electrophoresis and by PCR. As expected, the PCR product contained the full coding sequence plus 69 and $196 \mathrm{bp}$ of $5^{\prime}$ and $3^{\prime}$ UTRs respectively. The double-stranded cDNA was used successfully for creating a SSH cDNA library. The cDNA could also be useful for a number of other applications like cDNA library construction, EST analysis, RACE and Next Generation Sequencing (NGS).

Keywords: Bark tissue, cDNA, Bixa orellana, GAPDH, RNA.

Annatto dye is one of the important natural food colourant widely used in coloring dairy, confectionary, bakery and snacked foods (Akshatha and Giridhar, 2011). It finds use in dyeing leather, in pharmacy and in cosmetics. The annatto dye is obtained from the aril portion of seeds of Bixa orellana L. (Bixaceae). The major content of this is bixin, an apocarotenoid which is lipid soluble and a water soluble fraction norbixin. The annatto plant is native and very popular in South American countries, and now widely cultivated in different parts of the world. The average annatto dye with $2 \%$ bixin level is not acceptable in International market. It requires over $2.7 \%$ bixin containing seeds for export and trade. Under this context, augmentation of annatto dye in seeds of $B$. orellana $L$. is a necessity. The possibility of biotechnological methods for developing elite plants of $B$. orellana was investigated. In this report we describe a simple and effective protocol for the extraction of high quality RNA from bark 
tissue of woody stem of B. orellana plant and the method for converting this RNA to double strand cDNA. This cDNA is useful in an array of applications including gene expression analysis, construction of subtractive cDNA library (Diatchenko, 1996), qRT-PCR, RACE and for next generation sequencing.

\section{MATERIALS AND METHODS Plant Material}

Bark tissue from the woody stem of a 12 year-old Bixa orellana plant was used. The tissue was collected using a sterile scalpel blade and it was immediately frozen and stored in liquid nitrogen till used for RNA extraction.

\section{Total RNA isolation}

Total RNA was isolated using RNeasy plant mini kit (Qiagen, USA) following the procedure given in the user manual with slight modifications. All solutions used for total RNA isolations were prepared using Diethyl PyroCarbonate (Sigma, USA) treated MilliQ-water (Millipore, USA). All nondisposable plastic materials were treated with DEPC and autoclaved. The bark tissue frozen in liquid nitrogen was transferred to a prechilled pestle and mortar and immediately ground to a fine powder in liquid nitrogen. Around $100 \mathrm{mg}$ of the frozen ground tissue were transferred to $950 \mu \mathrm{l}$ of RLT buffer (Lysis buffer) in a microcentrifuge tube. The tissue was homogenized using a tissue homogenizer (Polytron PT 1200 E, Kinematica AG) for $2 \mathrm{~min}$ at full speed. The homogenized tissue lysate was passed through the silica membrane spin column provided with the RNeasy Plant Mini Kit (Qiagen, USA) and subsequently treated with DNase I (Qiagen, USA) to remove genomic DNA, according to the manufacturer's instructions. The integrity of the total RNA was analyzed using 1.2\% native agarose/EtBr gel electrophoresis. The concentration and purity of the total RNA were determined using a microvolume UV spectrophotometer (NanoDrop Technologies, USA).

\section{cDNA Synthesis}

First-strand cDNA was synthesized according to the SMARTer PCR cDNA synthesis kit. For this purpose $2.5 \mu \mathrm{l}$ of total RNA sample (about 500 ng), 3' SMART CDS primer II A (5'-AAG CAG GGT ATC AAC GCA GAG TAC $\mathrm{T}_{(30)} \mathrm{N}_{-1} \mathrm{~N}-3^{\prime}$ where $\mathrm{N}=\mathrm{A}, \mathrm{C}$, $\mathrm{G}$, or $\mathrm{T} ; \mathrm{N}_{-1}=A, \mathrm{G}$, or $\mathrm{C}$ ) were mixed and incubated first at $72^{\circ} \mathrm{C}$ for $3 \mathrm{~min}$ and then lowered to $42^{\circ} \mathrm{C}$ for $2 \mathrm{~min}$. $5 \mathrm{x}$ first strand buffer, DTT, dNTP mix and SMARTScribe Reverse Transcriptase were incubated at $42^{\circ} \mathrm{C}$ for $90 \mathrm{~min}$. The first strand reaction was terminated by heating the tubes at $70^{\circ} \mathrm{C}$ for ten minutes.

Amplification of cDNA by long distance polymerase chain reaction (LD-PCR)

$2 \mu \mathrm{l}$ of the first strand cDNA, deionized $\mathrm{H}_{2} \mathrm{O}$, Advantage 2 PCR buffer, dNTP mix, 5' PCR primer II A, and Advantage 2 polymerase mix were added into a pre-chilled microcentrifuge tube and Amplification of cDNA was done by long distance polymerase chain reaction (LDPCR). Then amplified using the following program; $95^{\circ} \mathrm{C} 1 \mathrm{~min} 24$ cycles of $95^{\circ} \mathrm{C} 15 \mathrm{sec}$, $6^{\circ} \mathrm{C} 30 \mathrm{sec}, 68^{\circ} \mathrm{C} 6 \mathrm{~min}$. $5 \mu \mathrm{l}$ of the PCR product was analyzed on agarose/EtBr gel electrophoresis. The cDNA was size fractionated and small DNA contaminants and unincorporated nucleotides were removed from the cDNA using CHROMA SPIN-1000 columns (Clontech) as per the manufacturer's instruction.

\section{Analysis of cDNA integrity}

$2 \mu l$ purified SMARTer cDNA was amplified using deionized $\mathrm{H}_{2} \mathrm{O}, 10 \mathrm{x}$ Advantage 2 PCR buffer, 50x dNTP mix, 5 pmol of forward and reverse primers, which were designed based on sequence available. 50x Advantage 2 polymerase mix using the following program: $94^{\circ} \mathrm{C} 4 \mathrm{~min} 30$ cycles of 
$94^{\circ} \mathrm{C} 30 \mathrm{sec}, 55^{\circ} \mathrm{C} 1 \mathrm{~min}, 72^{\circ} \mathrm{C} 1 \mathrm{~min}$. The PCR products were purified using the Qiaquick PCR purification kit (Qiagen, USA). The purified PCR product was ligated into pGEMT-Easy (Promega, USA) vector and transformed into competent JM109 E.coli cells. The transformed cells were spread on LB agar containing Xgal/IPTG/Ampicilin and incubated at $37^{\circ} \mathrm{C}$ overnight. Pure white colonies were randomly picked and inoculated into $2 \mathrm{ml}$ of LB broth with 100 $\mu \mathrm{g} / \mathrm{ml}$ concentration of ampicilin and grown at $37^{\circ} \mathrm{C}$ overnight in incubator shaker. Plasmid DNA was isolated from the overnight grown culture using Qiaprep Spin mini prep kit (Qiagen, USA). The purified plasmids were sequenced from the M13 region of the plasmids using Sanger's dideoxy method. The sequence of the GAPDH gene and deduced amino acid sequence was analyzed using DNASTAR (Madison, USA) and the homology search was performed using the BLAST program in the GenBank database.

\section{RESULTS AND DISCUSSION Isolation of total RNA}

The ratio of the $A_{260} / A_{280}$ for total RNA was 1.9 and around $20 \mu \mathrm{g}$ of total RNA was obtained from $100 \mathrm{mg}$ of the bark tissue. The total RNA showed two distinct bands corresponding to $28 \mathrm{~S}$ and $18 \mathrm{~S}$ ribosomal RNA bands on ethidium bromide stained 1.2\% agarose gel and the brightness of $28 \mathrm{~S}$ is as twice as that of 18S RNA (Figure 1). The results indicate that no degradation or contamination occurred during isolation and the total RNA isolated from B. orellana bark tissue was pure, integrated and suitable for cDNA synthesis.

cDNA synthesis and amplification of cDNA by long distance polymerase chain reaction (LD-PCR)

SMART technique is a novel and useful method for full length cDNA synthesis. Its unique characteristic is that it provides full-length cDNAs that preserve the complete $5^{\prime}$ terminal sequence of the mRNA. In this method, as the superiority of LD-PCR in cDNA synthesis, the amount of available RNA starting material needed is very small, which is significant for situations where the original sample supply is limited by difficult gaining samples. Using the SMARTer cDNA synthesis and long-distance PCR (LD-PCR) amplification strategy (Barnes 1994), it was possible to synthesize high quality cDNA from RNA of the woody bark tissue of $B$. orellana. Majority of the cDNA produced from LD-PCR was analyzed on a $1.2 \%$ agarose gel and a homogenous smear of PCR product ranging from 0.2 to $4 \mathrm{~Kb}$ in size was observed.

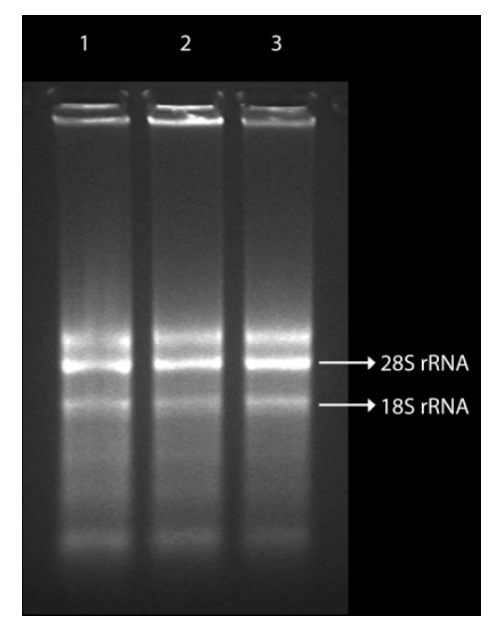

Figure 1: Total RNA isolated from 3 independent bark tissue of $B$. Orellana using the Qiagen RNeasy plant mini kit. Lane 1, 2 and 3: Total RNA with 2:1 ratio of $28 \mathrm{~S}$ rRNA and $18 S$ rRNA.

Subsequently the synthesized cDNA was purified by CHROMA SPIN 1000 column to remove short DNAs. Full length representation of cDNA population is the key for most of the functional genomic applications and in the present study we provide the efficient method of total RNA from bark tissue using the RNeasy plant mini kit. Synthesis of double stranded cDNA was achieved from minimum quantity of the 
starting material and the quality of the cDNA was assessed by cloning full length of coding region plus UTRs at terminal of the GAPDH gene (Figure 2). The Insilco analysis of the GAPDH gene revealed a complete coding region of $1015 \mathrm{bp}$ starting from the ATG start codon to TGA stop codon. In addition to this $69 \mathrm{bp}$ and $196 \mathrm{bp}$ of $5^{\prime}$ and 3' UTRs was found. The analysis of the GAPDH gene confirms that intact RNA was obtained and the cDNA synthesized contains the complete representation of the genes expressed.

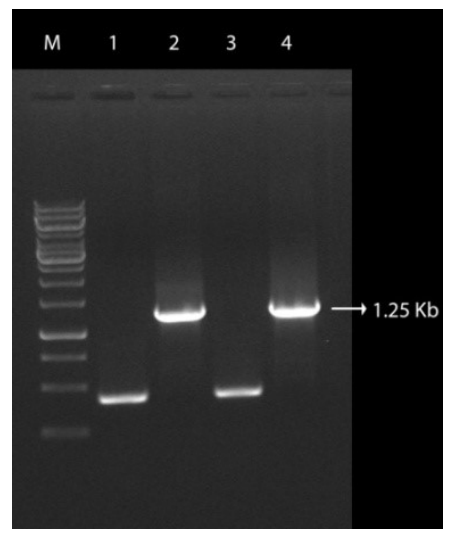

Figure 2: Analysis of the ds cDNA by PCR amplification. Lane $\mathrm{M}: 1 \mathrm{~Kb}$ marker (Fermentas) Lane 1, 3 and 2, 4: replicates of the partial glucanase gene $(0.4 \mathrm{~Kb})$ and full length of GAPDH gene $(1.25 \mathrm{~Kb})$.

\section{Conclusion}

For the first time we describe an effective protocol for total RNA isolation from bark tissue of B. orellana and synthesis of full length double stranded cDNA. We successfully used the cDNA for construction of a subtractive cDNA library by $\mathrm{SSH}$ technique (results not reported here). This
cDNA could be useful for an array of applications including gene expression analysis, isolation of full length genes, qRTPCR, RACE and for Next Generation Sequencing (NGS).

\section{References}

Akshatha V, Giridhar P, Ravishankar GA 2011. Morphological diversity in Bixa orellana L. and variations in annatto pigment yield. J Hort Sci Biotechnol 86: 319-324

Barnes WM. 1994. PCR amplification of up to 35-kb DNA with high fidelity and high yield from lambda bacteriophage templates. Proc. Nat. Acad. Sci. 91(6): 2216-2220

Diatchenko L, Lau YFC, Campbell AP, Chenchik A, Moqadam F, Huang B, Lukyanov K, Gurskaya N, Sverdlov ED, Siebert PD. 1996. Suppression subtractive hybridization: a method for generating differentially regulated or tissue specific cDNA probes and libraries. Proc. Natl. Acad. Sci. 93: 6025-6030.

Lee S, Jeon JS, Jung KH, An G. 1999. Binary vectors for efficient transformation of rice. J. Plant Biol. 42: 310-316.

Sambrook J, Russell DW. 2001. Extraction, purification and analysis of mRNA from eukaryotic cells. Molecular Cloning: a laboratory manual. Cold Spring Harbor Laboratory press. ISBN:0-87969-577-3. 1: 7.82 .

Sreenath HL, Prakash NS. 2006. Coffee. Plantation Crops 1. (Eds.) Parthasarathy VA, Chattopadyaya PK and Bose TK. NayaUdyog, Kolkata. ISBN 8185971978.149-264. 